Article

\title{
Minimising Non-Processing Energy Consumption and Tardiness Fines in a Mixed-Flow Shop
}

\author{
Chen Peng ${ }^{1}$, Tao Peng ${ }^{1} * \mathbb{D}$, Yi Zhang ${ }^{2}$, Renzhong Tang ${ }^{1}$ and Luoke Hu ${ }^{1}$ \\ 1 Institute of Industrial Engineering, School of Mechanical Engineering, Zhejiang University, \\ Hangzhou 310027, China; pcme@zju.edu.cn (C.P.); tangrz@zju.edu.cn (R.T.); 11125069@zju.edu.cn (L.H.) \\ 2 College of Information Science \& Electronic Engineering, Zhejiang University, Hangzhou 310027, China; \\ 21731108@zju.edu.cn \\ * Correspondence: tao_peng@zju.edu.cn; Tel.: +86-(0)571-8795-1145
}

Received: 2 November 2018; Accepted: 28 November 2018; Published: 3 December 2018

\begin{abstract}
To meet the increasingly diversified demand of customers, more mixed-flow shops are employed. The flexibility of mixed-flow shops increases the difficulty of scheduling. In this paper, a mixed-flow shop scheduling approach (MFSS) is proposed to minimise the energy consumption and tardiness fine (TF) of production with a special focus on non-processing energy (NPE) reduction. The proposed approach consists of two parts: firstly, a mathematic model is developed to describe how NPE and TF can be determined with a specific schedule; then, a multi-objective evolutionary algorithm with multi-chromosomes (MCEAs) is developed to obtain the optimal solutions considering the NPE-TF trade-offs. A deterministic search method with boundary (DSB) and a non-dominated sorting genetic algorithm (NSGA) are employed to validate the developed MCEA. Finally, a case study on an extrusion die mixed-flow shop is performed to demonstrate the proposed approach in industrial practice. Compared with three traditional scheduling approaches, the better performance of the MFSS in terms of computational time and solution quality could be demonstrated.
\end{abstract}

Keywords: energy consumption; scheduling approach; mixed-flow shop; multi-objective optimisation; tardiness fine

\section{Introduction}

Manufacturing accounts for about 25\% of global energy consumption [1]. Energy Information Administration (EIA) reports that $90 \%$ of the processing electricity in industries was consumed by manufacturing [2]. Energy-efficient manufacturing, which can be implemented through production facility improvements, has been encouraged [3] but this increases the financial burden to build or purchase new facilities. Scheduling has been approved to be an effective and economic tool to reduce the manufacturing energy consumption of production facilities (MEPF) [4].

MEPF can be divided into the non-processing energy (NPE) and processing energy (PE) consumption of production facilities. NPE represents the energy consumption of production facilities during the non-processing phase and it is the integral of idle power over the relevant idle time [5]. $\mathrm{PE}$ represents the energy consumption of production facilities during the processing phase, which is associated with the processing power and processing time of the facilities. Research indicates that the non-processing power reaches up to $30 \%$ of the processing power [6]. However, the processing time of production facilities is normally shorter than their non-processing time. For example, Wiendahl investigated six industries and found that the processing time only accounted for $15 \%$ of the total manufacturing time [7]. Therefore, the NPE can be large.

Scheduling has been proven to be crucial in manufacturing and it plays an important role for companies to meet due dates committed to by customers [8]. Meanwhile, the existing literature 
suggests that the NPE can be effectively reduced by scheduling at the production plan stage in job shops [9] and in flow shop environments [10], while application scenarios in mixed-flow shop environment are limited.

The mixed-flow shop environment is critical to quickly respond to the diversified demand of customers in today's manufacturing, such as an extrusion die workshop. Compared with the traditional flow shop, the jobs in mixed-flow shops have standard as well as customised processes. A standard process is defined as a process with the same operations among multiple jobs in which all the jobs need to be executed on the machines with the same route during the standard process. A customised process is defined as the specific operations of jobs, operations that are applied for processing the personalised parts of the products. For the extrusion die making industry, the diversification is obvious, therefore, building a mixed-flow shop environment is necessary. Extrusion dies are customised for various Aluminium profiles. Different dies share some standard processes, such as cylindrical turning, end milling, and other basic processing, while the manufacturing of customised holes in dies is diverse. Furthermore, a real-operating extrusion die workshop needs to produce more than thirty thousand types of dies in one year, while the daily average yield is only seventy. The current productivity cannot meet the requirement and the tardiness fine (TF) is severe. The TF is the compensation (financial or other forms) to clients caused by the tardiness and the amount of compensation per unit time is based on the contracts. Besides, the idle machines are common in workshops. Scheduling has been proven to be an effective method to guarantee punctual deliveries [11] and reduce the NPE consumed by machines while waiting for jobs [12]. To minimise the NPE and TF in a mixed-flow shop, this paper formulates a multi-objective optimisation problem and proposes a mixed-flow shop scheduling approach (MFSS) to solve it. The MFSS is a scheduling approach aimed at minimising the NPE and TF, and it contains two parts: a mathematic model describing how the NPE and TF are influenced by a specific schedule, and a multi-objective evolutionary algorithm with multi-chromosomes (MCEAs) to obtain the optimal solutions considering the NPE-TF trade-offs.

When employing a scheduling approach to optimise the NPE and TF in mixed-flow shops, there are several difficulties: (1) the process routes can be different, thus, uncertainties for production facilities exist when selecting the next job to process with considerations toward the NPE and TF; (2) the job sequence on each facility can be different, thus, the computation scale is larger than that of a typical flow shop with the same amount of jobs and machines; (3) a trade-off between the NPE and TF should be considered because there exists a conflict between the NPE and TF. For example, when the tardiness fine is minimised, the NPE of the production facilities may increase due to the continuous running of the machines to ensure production readiness. The proposed approach to address the above difficulties is the main contribution of this paper.

NPE is first characterised in the mixed-flow shop environment and its relationship with TF is then analysed. The relationship between the scheduling scheme and the TF and NPE are reflected, and then a TF-NPE bi-objective optimisation model is developed. The bi-objective optimisation in this research is to achieve optimal trade-offs in completing a production task by properly scheduling the jobs. The MCEA is proposed to search for the Pareto optimum. An optimal solution represents a scheduling scheme that results in the optimal trade-offs between the two objectives. Through a case study, the developed models and optimisation approaches are demonstrated, compared, and discussed.

In the remainder of this paper, the literature review is presented in the following section. The description of the research problem and the bi-objective model are given in Section 3. In Section 4, the working procedure of the MCEA for solving this optimisation problem is described. A case study is conducted to demonstrate the applicability and effectiveness of the developed approach in Section 5. Finally, a brief summary and future works are given in Section 6.

\section{Related Work}

An increasing amount of energy-aware scheduling research has been conducted intensively in the recent twenty years, from which four typical scenarios can be found: the single machine, parallel 
machine, flow shop, and job shop scenarios. In single machine scheduling, Mehmet et al. provided a methodology for decision-makers to choose the most efficient scheduling with the appropriate energy consumption of a single machine and an efficient genetic algorithm was developed [13]. Shrouf et al. presented a scheduling method to minimise the energy consumption costs whilst considering the variable energy prices during the day [14]. Yin et al. reduced the total earliness/tardiness cost and energy consumption of a single machine by controlling the processing times and turn off/on [15]. In parallel machines scheduling, Zhantao Li considered the unrelated parallel scheduling problem in the background of big data, with the total tardiness and energy consumption as two objectives. Ten heuristic algorithms were developed to solve the mathematic model, and their performance was tested by designing computational experiments [16]. Moon et al. [17] developed a method to optimise both the makespan of production and time-dependent energy costs of the unrelated parallel machine. Ding et al. [18] studied the unrelated parallel machine scheduling problem in a time-of-use pricing scheme, aiming to minimise the total electricity cost. However, the energy-aware scheduling research on a single machine and parallel machines is not sufficient to solve the problem of mixed-flow shops because the NPE of a machine is affected by other machines in its involved processes. In the mixed-flow shop, jobs and machines should be considered systematically.

For flow shop and job shop scheduling, a large amount of multi-objective optimisation research can be referred to. Lu et al. formulated a mathematic model concerning the transportation and sequence-dependent setup stage and proposed a hybrid multi-objective backtracking search algorithm to solve the model [10]. Zhang et al. proposed a time-indexed integer programming formulation to optimise the electricity cost and the $\mathrm{CO}_{2}$ emissions at the same time [19]. Liu et al. developed a model that minimises the total non-processing energy consumption and total weighted tardiness in a job shop, and the turn off/on strategy was employed as an energy saving approach [20]. Zhang et al. solved the bi-objective, total weighted tardiness, and energy consumption in the job shop scheduling problem with a multi-objective genetic algorithm including the local improvement strategies and compared its performance to that of NSGA-II [21]. Chen et al. studied the energy consumption reduction in Bernoulli serial lines with finite buffers and machines through the effective scheduling of machine startups and shutdowns [4].

The actual production is not limited to traditional job shops or flow shops. Energy-aware scheduling research is carried out in more complex production scenarios. Tang et al. and Dai et al. modified the particle swarm optimisation algorithm and genetic-simulated annealing algorithm to get the optimal schedule in flexible flow shops [22,23]. Li et al. proposed a scheduling approach based on Petri net models and a genetic algorithm to reduce the total energy consumption in flexible manufacturing systems [24]. Mouzon et al. investigated the impact of dispatching rules on reducing the energy consumption of manufacturing equipment [25]. Zhang et al. presented a novel approach of dynamic rescheduling in flexible manufacturing systems, concerned with energy consumption and schedule efficiency [26]. Liu et al. introduced a mixed-integer nonlinear programming model for the hybrid flow shop scheduling problem by minimising the energy consumption and setting up a constraint to require all the jobs to be delivered on time, though this constraint can sometimes not be satisfied in practical production [27]. Tong et al. and Li et al. analysed the production characteristics of forging shops and welding shops, respectively, and put forward corresponding energy-saving scheduling schemes $[28,29]$. As in the aforementioned study in complex production scenarios, energy consumption can be reduced observably by the schedule. However, the models or algorithms of one scheduling approach cannot be universally applied to other types of scenarios.

According to the literature reviewed, energy-aware scheduling research in various scenarios has been conducted. While the research on diversified customised production scenarios is rarely considered, Zhou et al. described the characteristics of multi-varieties and the small-batch production scheduling mode and proposed an improved genetic annealing algorithm to shorten the production cycle, maximise the resource utilization rate, etc. [30]. Huang et al. designed a polynomial-time dynamic programming algorithm to minimise the makespan in a flow shop for mass customization [31]. 
Wang et al. established a Petri net based real-time model for hybrid flow shops to satisfy the small-batch customised production [32]. Huang et al. discussed the re-scheduling problems of a mixed-line flow shop and a drum-buffer-rope technique is applied to minimise the longest tardiness for the customers' orders [33]. The research articles above studied the scheduling problem in customised production scenarios with some of them being the mixed-flow shop scenario. However, the makespan and resource allocation are the main objectives and energy consumption is rarely concerned.

Based on the above discussion, the energy-aware scheduling research in mixed-flow shops is limited and the existing scheduling approaches in the other types of workshops cannot be applied in this environment because each scenario has its own features, including mixed-flow shop scenarios. These features should be taken into consideration when proposing a scheduling approach. In a mixed-flow shop, both standard processes and customised processes exist among the jobs' routes. As a result, the scheduling models and algorithms in a mixed-flow shop are more complex than in a traditional flow shop. To bridge such a gap, a scheduling approach specifically for the mixed-flow shop to minimise TF and NPE is introduced in the following sections.

\section{Problem Statement}

The problem addressed in this paper is a mixed-flow shop scheduling problem with NPE and TF objectives. In this scenario, $n$ jobs $\left\{J_{p}\right\}_{p=1}^{n}$ are to be processed on $m$ machines $\left\{M_{k}\right\}_{k=1}^{m}$ in sequence and a job contains a batch of the same workpieces. Each job $J_{p}$ has an established process route $\left\{O_{p}^{j}\right\} . O_{p}^{j}$ is equal to $k$ in number and all the jobs flow according to the ascending machine numbers. $t_{p}^{k}$ denotes the processing time of $J_{p}$ on $M_{k}$. Each job has a release time $r_{p}$ and a due date $d_{p}$. In manufacturing, a feasible job sequence $S_{k}=\left\{J_{k_{i}}\right\}_{i=1}^{r_{k}}$ will be arranged on $M_{k}$, where $J_{k_{i}}$ is the $i$-th job processed on $M_{k}$ and $r_{k}$ is the total number of jobs using $M_{k}$. In addition, the jobs in $\left\{J_{k_{i}}\right\}$ have a one-to-one relationship with those jobs to be processed on $M_{k}$. An example of the directed graph of a mixed-flow shop is illustrated in Figure 1. The idle time and NPE vary along with the scheduling approaches for the jobs' processing time. Therefore, the key issue is to find the job permutation strategy for each machine that minimises the NPE as well as TF.

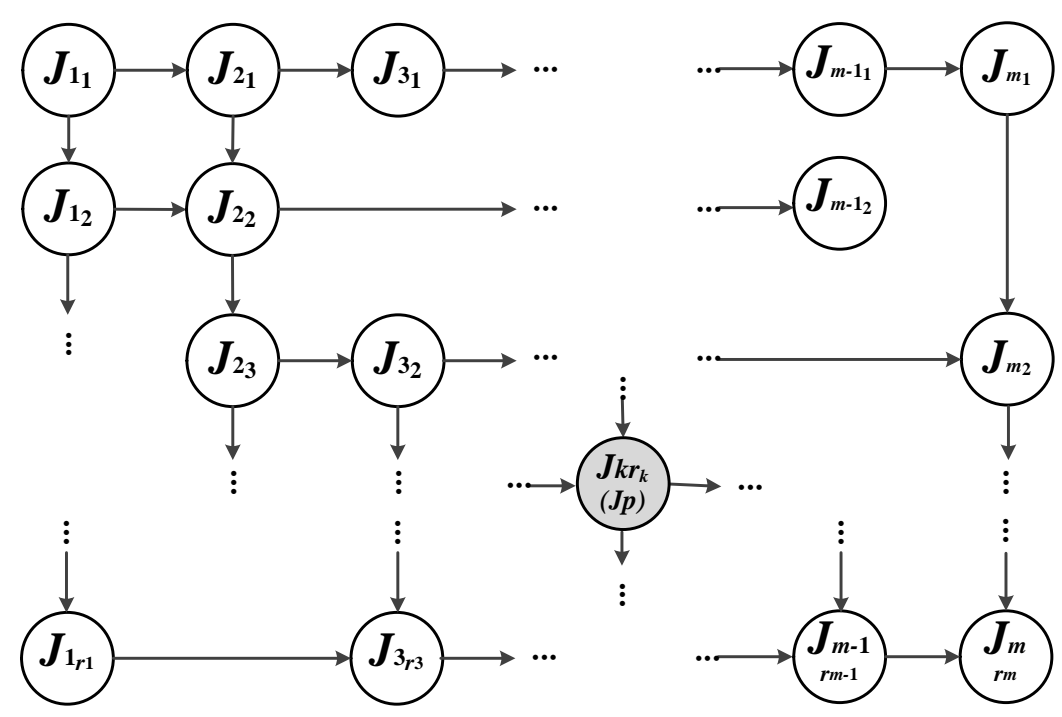

Figure 1. An example of the directed graph of a mixed-flow shop.

NPE is the integration of the machines' idle power in their idle time, respectively. To reduce the NPE, an optimal job sequence can be adopted, and this planned schedule should ensure the due dates of all jobs. However, the relationship between minimising NPE and ensuring the due date is not positive. In other words, a conflict exists between them. The following scheduling situation is taken as an example (see Figure 2). When $J_{5}$ on $M_{3}$ and $M_{4}$ is arranged to $B$, the other jobs' arrangements are 
not affected. The TF of other jobs and the NPE of $M_{1}, M_{2}$, and $M_{5}$ are unchanged while the replaced job's TF decreases and the NPE values of $M_{3}$ and $M_{4}$ increase. As a result, the total TF decreases and the NPE rises.

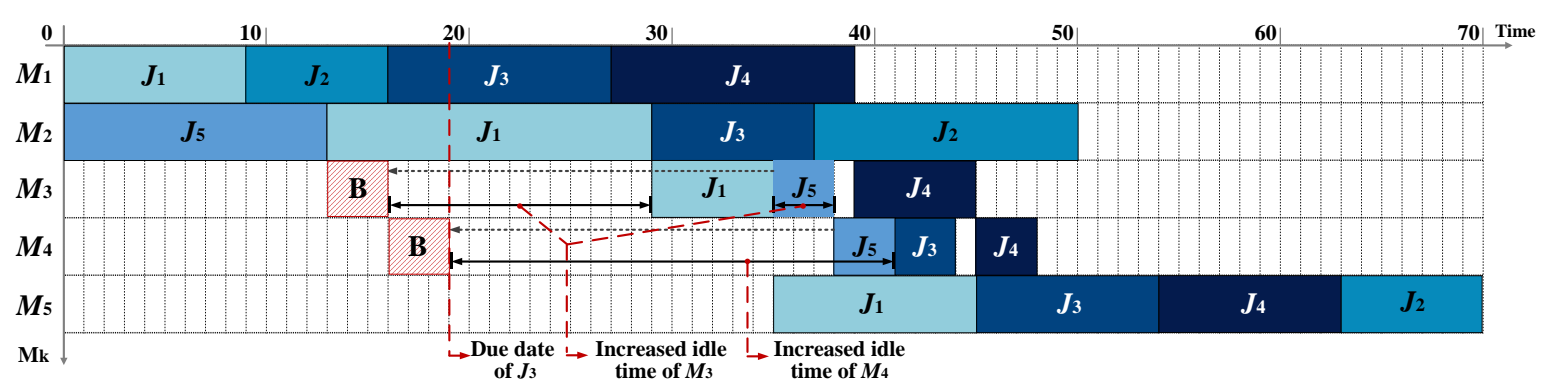

Figure 2. An exemplifying situation for the conflict between NPE and TF.

Hence, a multi-object mathematic model to get an optimal schedule strategy is developed. The assumptions are as follows:

(1) All the machines run from the first workpiece and come without the priority among the jobs;

(2) The release time of jobs is the same;

(3) One machine is able to process one workpiece simultaneously;

(4) All processes are non-preemptive and no machine needs to be used twice for one job;

(5) The processing time of each job is generated as a statistical average value;

The process route, processing time, release time, and due date of each job and the idle power of the machines are given. Only when a job is completed before the due date is the order considered to be completed punctually without paying a tardiness fine. The value of the tardiness fine per unit time has been determined by the contracts.

The multi-objective model aims to find the most energy-saving scheduling scheme of jobs with the minimum overdue fine. The multi-objective model Multi aims to minimise the NPE and TF at the same time:

$$
\text { Multi }=\text { Minimise }\{\operatorname{TF}(s), \operatorname{NPE}(s)\}
$$

The model has two parts. One part is the NPE model. NPE can be minimised by shortening the non-processing time of the machines caused by queuing. The non-processing time is the idle time when the previous job on a machine has finished and the next job has yet arrived. The other part is the TF model. The total TF is a sum of fines in all considered jobs, which is affected by the job makespan, due date, and penalty mechanism.

For $n$ jobs $\left\{J_{p}\right\}_{p=1}^{n}$ to be processed on $m$ machines, $\left\{M_{k}\right\}_{k=1}^{m}$ in a mixed-flow shop, the job sequence $S_{k}$ on each $M_{k}$ cannot be the same. Given a feasible scheduling scheme $s$, the total TF of all jobs in this scheduling can be calculated as

$$
\operatorname{TF}(s)=\sum_{p=1}^{n} T_{p} \times F_{p}
$$

where $T_{p}$ and $F_{p}$ are the tardiness time and tardiness fine per unit time of $J_{p}$, respectively. The total NPE of all machines can be calculated as

$$
\operatorname{NPE}(s)=\sum_{k=1}^{m} P_{k}^{\text {idle }} \times t_{k}^{\text {idle }}
$$

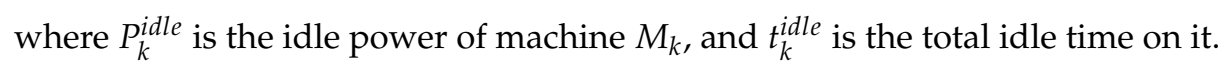

The detailed mathematic models are shown in the appendix. To achieve the optimal scheduling schemes of minimising the TF and NPE with the model, a feasible algorithm needs to be adopted. 


\section{Multi-Objective Optimisation}

To obtain the optimal job sequence, heuristic or deterministic algorithms can be employed to solve the model. The global optima can be acquired by the deterministic algorithm. However, its computation burden is heavy. Although heuristic algorithms can find a feasible sequence within a short time, it normally traps the sequence into the local optimum. To obtain a high-quality solution within a tolerant computation time, a multi-objective evolutionary algorithm with multi-chromosomes (MCEsA) is proposed. Besides, a deterministic search method with boundaries is used to validate the accuracy of the MCEA.

MCEA is designed based on a genetic algorithm, which is a robust meta-heuristic that imitates the process of natural selection. As stated in the aforementioned problem, the job sequence and the job quantity to be processed on each machine are different. To code the scheduling scheme feasibly, a two-dimensional array is proposed as an individual. Each row vector, regarded as a chromosome, represents a job processing sequence on a machine. One individual represents a feasible scheduling scheme s and can be expressed as follows:

$$
\text { Individual : }\left\{\begin{array}{c}
{\left[J_{1_{1}}, J_{1_{2}}, J_{1_{3}}, \ldots J_{1_{v}}, \ldots J_{1_{r(1)}}\right]} \\
{\left[J_{2_{1}}, J_{2_{2}}, J_{2_{3}}, \ldots J_{2_{v}}, \ldots J_{2_{r(2)}}\right]} \\
\ldots \\
{\left[J_{k_{1}}, J_{k_{2}}, J_{k_{3}}, \ldots J_{k_{v}}, \ldots J_{k_{r(k)}}\right]}
\end{array}\right\}
$$

where $J_{k_{v}}$, regarded as a gene, denotes the $v$-th job processed on $M_{k} . r(k)$ is the total amount of jobs on the machine. The flow chart of MCEA is shown in Figure 3.

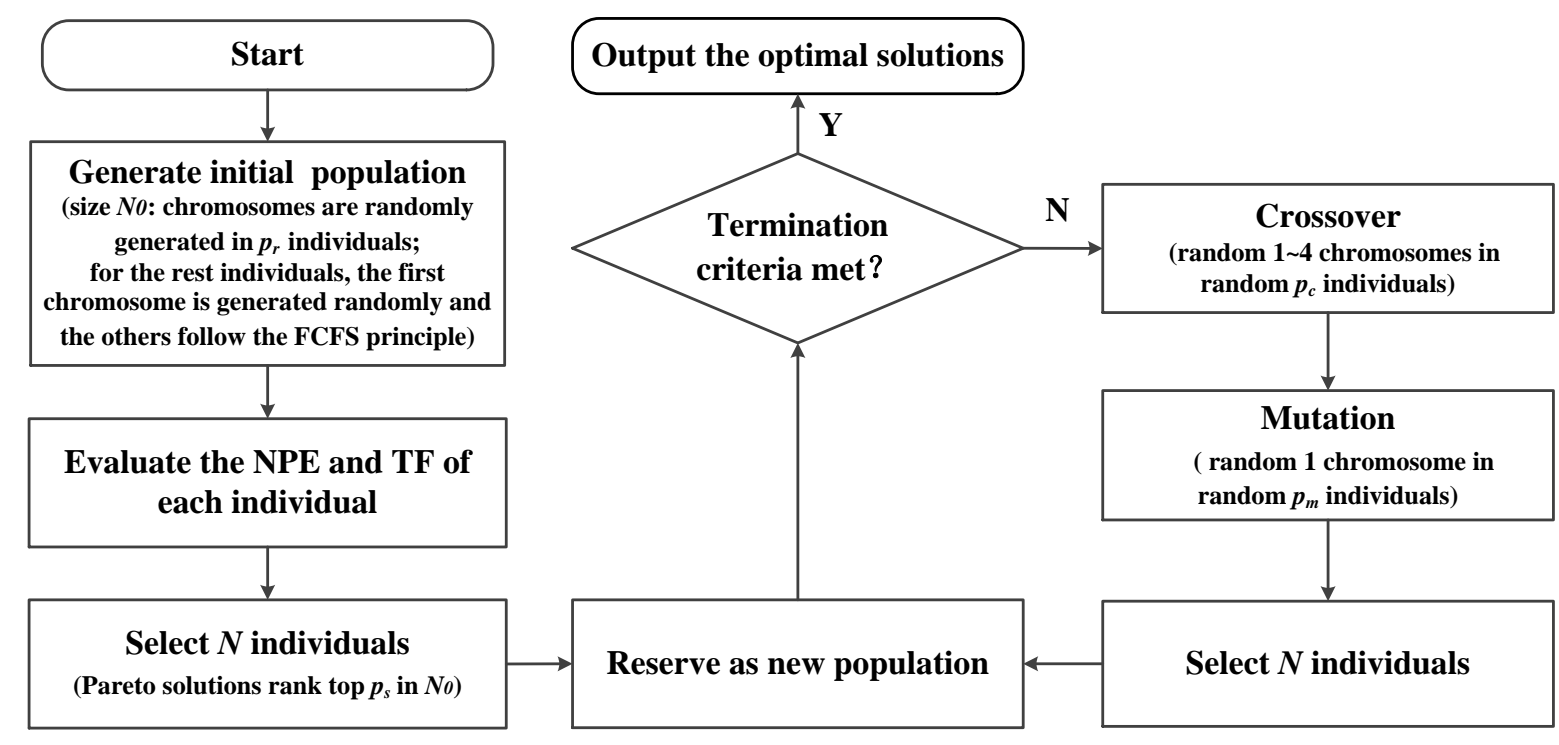

Figure 3. The flowchart of the MCEA.

First, the chromosome's coding method and fitness function should be given. In the MCEA, a gene, a chromosome, and an individual represent a certain job, a job sequence, and a scheduling scheme $s$, respectively. Second, the initial population with $N_{0}$ individuals is generated. Two principals are applied in generating the initial population: one is that all the chromosomes in individuals are generated randomly; the other one is that the first chromosome in the individual is randomly generated and the other chromosomes follows; first come first served. The $p_{r}$ of the $N_{0}$ individuals are generated by the first principal and the rest are generated by the second one. The initial population is selected by the fitness function, where fitness $1=N P E(s)$ and fitness $2=T F(s)$. Then $N_{0}$ individuals whose Pareto solutions rank the top $p_{s}$ in $N_{0}$ as a new population are reserved. Third, the children population 
is produced by crossovers and mutations: (1) Crossover: exchanging a random 1-4 chromosomes between two individuals. The crossover rate is $p_{c}$. (2) Mutation: modifying 1 random chromosome in the individuals. The mutation rate is $p_{m}$. Fourth, the next generation is selected from the combination of the individual parent and children by evaluating the fitness function. Once the iteration achieves $G$, the process ceases and outputs the non-dominant optimal solutions. If the termination criteria are not met, step four and step five repeat once more. In addition, all of the random selections are created by the roulette wheel approach.

The Deterministic Search Method with Boundaries (DSB) is an improved enumeration algorithm which can validate the accuracy of the MCEA. DSB traverses all the paths based on a depth-first search with an initial bound which is a set of non-dominant Pareto solutions output by MCEA. In the searching process, new schemes with better Pareto solutions replace the inferior ones within the boundaries and the else-scheduling schemes are pruned. When the traversal is completed, the Pareto solutions within the latest updated boundaries are reported as the global optimum.

\section{Case Studies and Discussion}

Aluminium profiles are customised products. To meet the diversified aluminium profiles, the matched dies need to be manufactured. The production of extrusion dies is carried out through multi-specifications and small batch manufacturing. Extrusion dies are shown in Figure 4. Both standard processes and customised processes exist among the different dies' processing routes and the extrusion die workshop is a typical mixed-flow shop. Three cases in the extrusion die workshop are studied in this paper to verify the performance of the MCEA and demonstrate the MFSS, respectively. Algorithms are developed on the Visual Studio 2013 software with the $\mathrm{C}++$ programming language. The computing platform is an Intel (R) Core (TM) i5-4210U CPU with a $1.70 \mathrm{GHz}$ frequency; $8.00 \mathrm{~GB}$ of RAM; Windows 10 (64 bit) operating system.

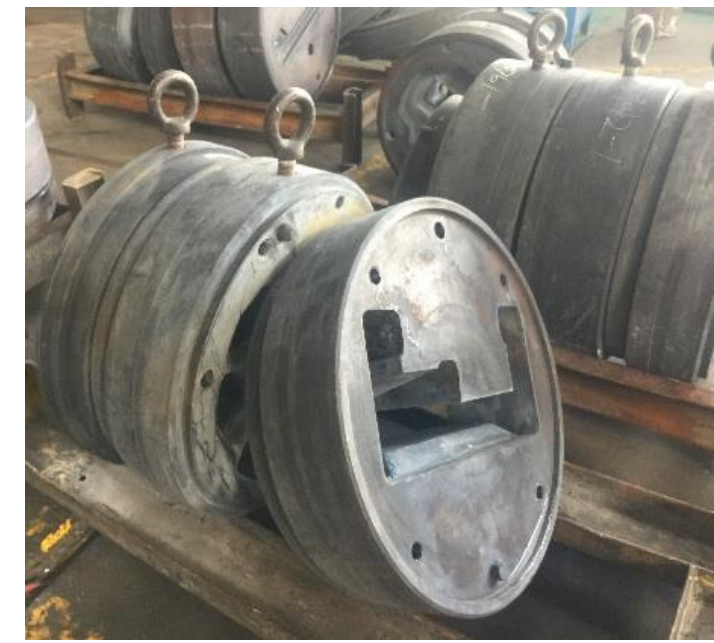

(a)

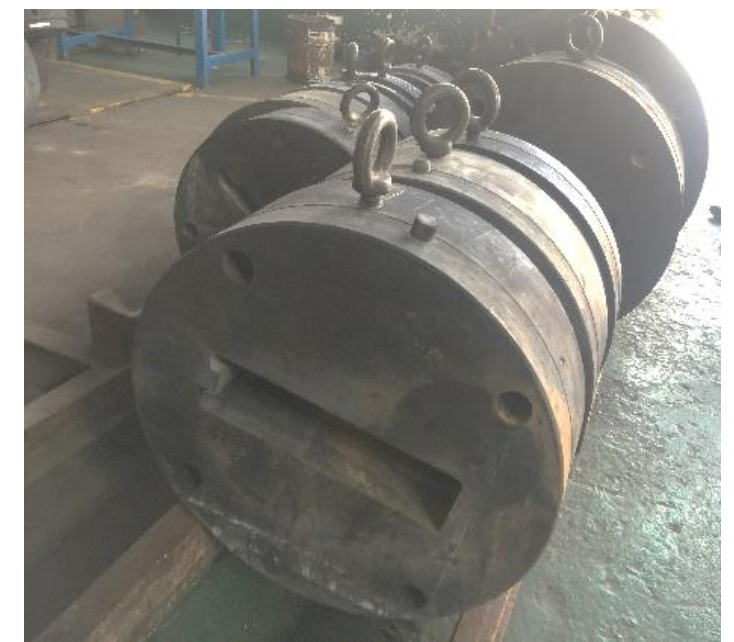

(b)

Figure 4. Extrusion dies with customised holes for different aluminium profiles (a) and (b).

\subsection{Comparison of the MCEA with the DSB}

To evaluate the performance of the MCEA, two cases including 4 jobs $\times 5$ machines and 5 jobs $\times 5$ machines are tested, respectively. The production data are generated based on the production tasks in an extrusion die workshop; see Tables 1 and 2. 
Table 1. The basic data in case 1 ( 4 jobs $\times 5$ machines).

\begin{tabular}{cccccccc}
\hline \multirow{2}{*}{ Job } & \multirow{2}{*}{ Due Date } & \multirow{2}{*}{$\begin{array}{c}\text { Tardiness Fine } \\
\text { per Unit Time }\end{array}$} & $\boldsymbol{M}_{1}$ & $\boldsymbol{M}_{2}$ & $\boldsymbol{M}_{3}$ & $\boldsymbol{M}_{4}$ & $\boldsymbol{M}_{5}$ \\
\cline { 4 - 8 } & & 2 & 9 & $\boldsymbol{P}_{2}^{\text {idle }}=3.2$ & $\boldsymbol{P}_{3}^{\text {idle }}=2.8$ & $\boldsymbol{P}_{4}^{\text {idle }}=2.1$ & $\boldsymbol{P}_{5}^{\text {idle }}=3.7$ \\
\hline$J_{1}$ & 50 & 3 & 11 & 8 & 6 & - & 10 \\
$J_{2}$ & 39 & 1 & 8 & 13 & - & 3 & 9 \\
$J_{3}$ & 32 & 5 & 12 & - & 6 & 3 & - \\
$J_{4}$ & 46 & & & & & 3 & 9 \\
\hline
\end{tabular}

Table 2. The basic data in case 2 ( 5 jobs $\times 5$ machines).

\begin{tabular}{cccccccc}
\hline \multirow{2}{*}{ Job } & \multirow{2}{*}{ Due Date } & \multirow{2}{*}{ Tardiness Fine } & $\boldsymbol{M}_{1}$ & $\boldsymbol{M}_{2}$ & $\boldsymbol{M}_{3}$ & $\boldsymbol{M}_{4}$ & $\boldsymbol{M}_{5}$ \\
\cline { 4 - 8 } & & & $\boldsymbol{P}_{1}^{\text {idle }}=3.2$ & $\boldsymbol{P}_{2}^{\text {idle }}=3.5$ & $\boldsymbol{P}_{3}^{\text {idle }}=2.8$ & $\boldsymbol{P}_{4}^{\text {idle }}=2.1$ & $\boldsymbol{P}_{5}^{\text {idle }}=3.7$ \\
\hline$J_{1}$ & 41 & 2 & 8 & 6 & 8 & - & 5 \\
$J_{2}$ & 39 & 1 & 7 & 3 & - & 6 & 10 \\
$J_{3}$ & 46 & 5 & 11 & 8 & - & 3 & 9 \\
$J_{4}$ & 35 & 3 & 9 & 7 & 3 & 5 & - \\
$J_{5}$ & 52 & 4 & 12 & 5 & 6 & 3 & 9 \\
\hline
\end{tabular}

The results of DSB and MECA in the two cases are shown in Figure 5. To assess the solution quality of MCEA, the hypervolume indicator is applied [34,35]. The maximum value of the NPE and TF in the results is taken as the reference point's horizontal and vertical coordinates, respectively [36]. The results of one test in 4 jobs $\times 5$ machines is reflected in Figure 6 and the point $R$ is the reference point. The area enclosed in red straight lines represents the dominated subspaces of solutions in DSB. The smaller area enclosed in blue dotted lines represents the dominated subspaces of the solutions in MCEA.

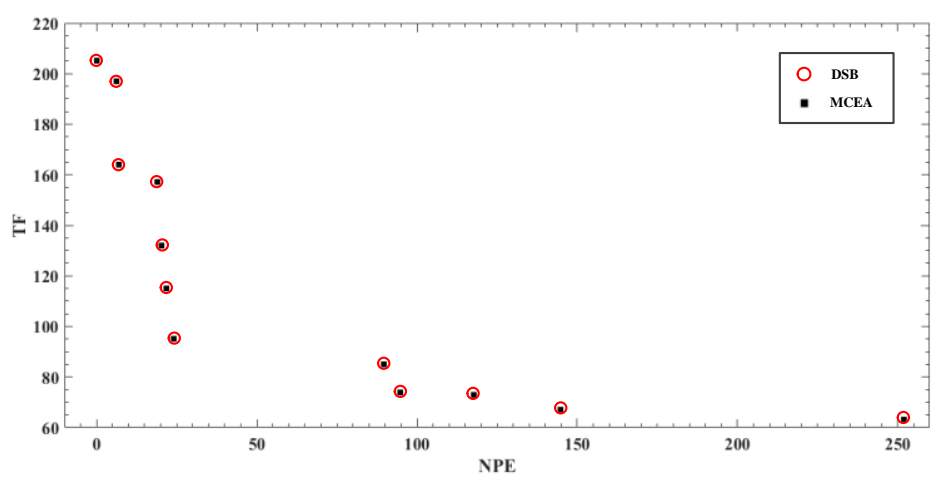

(a)

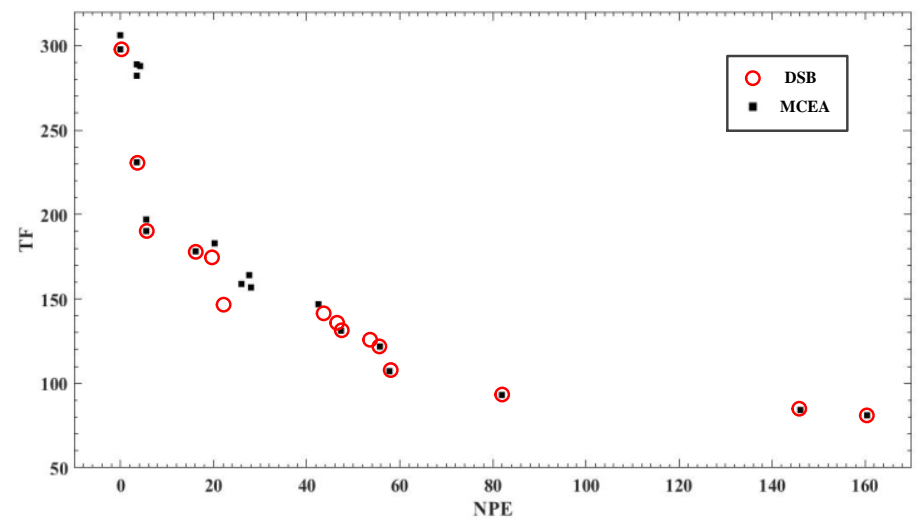

(b)

Figure 5. The results of DSB and MCEA, (a) in 4 jobs $\times 5$ machines, (b) in 5 jobs $\times 5$ machines. 


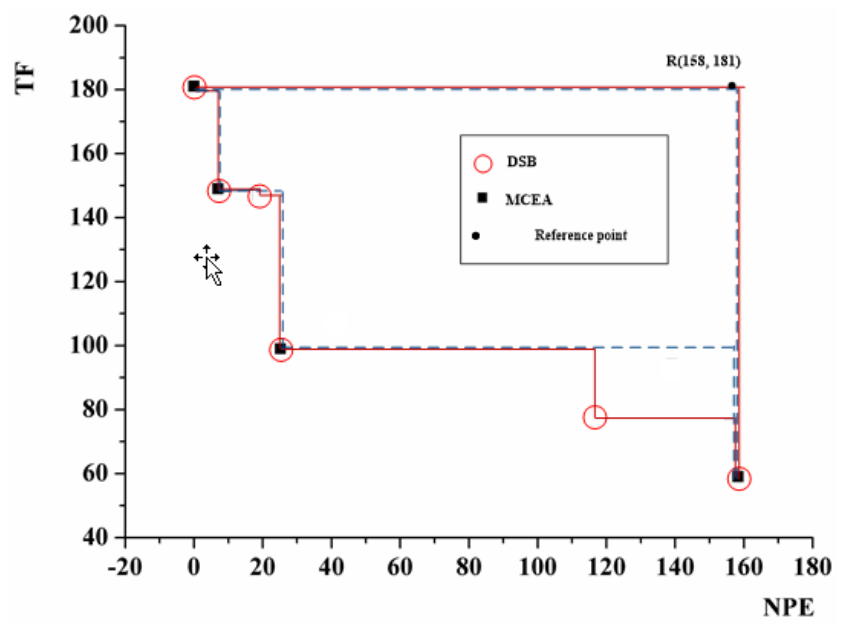

Figure 6. The calculation method schematic diagram of the hypervolume indicator.

The hypervolume indicator can be calculated as the dominated subspaces ratio of MCEA to DSB. The average hypervolume indicators of MCEA in case 2 and 3 are $99.791 \%$ and $97.950 \%$, respectively, while the hypervolume indicator of the MCEA varies with the cases because of the randomness of this algorithm. The larger the case, the more indeterminate the results of the algorithm. For MCEA, the global optimal solutions cannot be guaranteed as the DSB. The feasibility of MCEA in a larger case will be discussed in Section 5.3. Meanwhile, in actual practice, the computation time of the algorithms is also considered. In MCEA, the average computation time of the tests under 5 jobs $\times 5$ machines is $5.976 \mathrm{~s}$. While in DSB, the average computation time of the tests under 5 jobs $\times 5$ machines is $553.869 \mathrm{~s}$. Once the number of jobs or machines increased, the computing burden is heavier and the runtime of DSB will be over one hour, which cannot meet the real-time scheduling demand. Thus, when the scheduled task is larger than 5 jobs $\times 5$ machines, the MCEA could be employed to find the optimal scheduling schemes. Otherwise, both MCEA and DSB are capable.

\subsection{Application of the MFSS}

To demonstrate the MFSS, a case concerning 12 jobs and 5 machines in an extrusion die mixed-flow shop is selected. MFSS consists of the mathematic models and MCEA. The due date and tardiness fine data are randomly set. Each job contains a batch of the same parts and all the jobs are released at the same time. The effectiveness of the MFSS is demonstrated based on the case, as follows. The basic data of the case are listed in Table 3.

Table 3. The basic data in case 3 ( 12 jobs $\times 5$ machines).

\begin{tabular}{|c|c|c|c|c|c|c|c|}
\hline \multirow{2}{*}{ Job } & \multirow{2}{*}{ Due Date } & \multirow{2}{*}{$\begin{array}{l}\text { Tardiness Fine } \\
\text { per Unit Time }\end{array}$} & $M_{1}$ & $M_{2}$ & $M_{3}$ & $M_{4}$ & $M_{5}$ \\
\hline & & & $P_{1}^{i d l e}=5.2$ & $P_{2}^{i d l e}=3.5$ & $P_{3}^{\text {idle }}=2.8$ & $P_{4}^{\text {idle }}=2.1$ & $P_{5}^{\text {idle }}=3.7$ \\
\hline$J_{1}$ & 106 & 5 & 9 & 12 & 6 & 9 & 6 \\
\hline$J_{2}$ & 113 & 7 & 6 & 10 & 3 & 6 & - \\
\hline$J_{3}$ & 120 & 4 & 7 & 8 & 5 & 8 & 5 \\
\hline$J_{4}$ & 147 & 5 & 9 & 9 & 7 & 11 & 6 \\
\hline$J_{5}$ & 132 & 9 & 8 & 6 & 8 & - & 5 \\
\hline$J_{6}$ & 122 & 6 & 7 & 9 & - & 10 & 4 \\
\hline$J_{7}$ & 134 & 8 & 9 & 8 & 6 & 12 & 7 \\
\hline$J_{8}$ & 102 & 9 & 8 & 10 & - & 7 & - \\
\hline$J_{9}$ & 168 & 8 & 5 & 9 & 4 & 10 & 6 \\
\hline$J_{10}$ & 153 & 7 & 9 & 7 & 3 & 8 & 4 \\
\hline$J_{11}$ & 174 & 9 & 8 & 12 & 4 & - & 5 \\
\hline$J_{12}$ & 158 & 5 & 6 & 10 & - & 9 & 3 \\
\hline
\end{tabular}


After fine turning, the parameters in MCEA are as follows: the initial population size $N_{0}=1000$, population size $N=1000$, crossover rate $p_{c}=0.8$, mutation rate, $p_{m}=0.15$, and iteration $=1000$. A set of Pareto solutions is generated at the end of each run. One solution contains four parts: the scheduling schemes, TF, NPE, and a Gantt chart. A solution among the Pareto sets is chosen to verify the correctness of the model and algorithm. The details of the solution are shown in Table 4 and Figure 7. The start and completion time of each job and the idle time of each machine are visible in the Gantt chart.

Table 4. The job sequences, NPE and TF, of the example solution.

\begin{tabular}{cc}
\hline Machine & Job Sequence \\
\hline$M_{1}$ & $J_{5}-J_{4}-J_{8}-J_{7}-J_{3}-J_{2}-J_{9}-J_{12}-J_{6}-J_{1}-J_{11}-J_{10}$ \\
$M_{2}$ & $J_{4}-J_{5}-J_{8}-J_{3}-J_{9}-J_{12}-J_{2}-J_{7}-J_{1}-J_{6}-J_{11}-J_{10}$ \\
$M_{3}$ & $J_{9}-J_{4}-J_{3}-J_{2}-J_{7}-J_{1}-J_{5}-J_{11}-J_{10}$ \\
$M_{4}$ & $J_{9}-J_{8}-J_{2}-J_{4}-J_{7}-J_{6}-J_{3}-J_{12}-J_{10}-J_{1}$ \\
$M_{5}$ & $J_{5}-J_{6}-J_{11}-J_{3}-J_{9}-J_{4}-J_{12}-J_{7}-J_{1}-J_{10}$ \\
& $\mathrm{NPE}=92.3 \mathrm{TF}=715$ \\
\hline
\end{tabular}

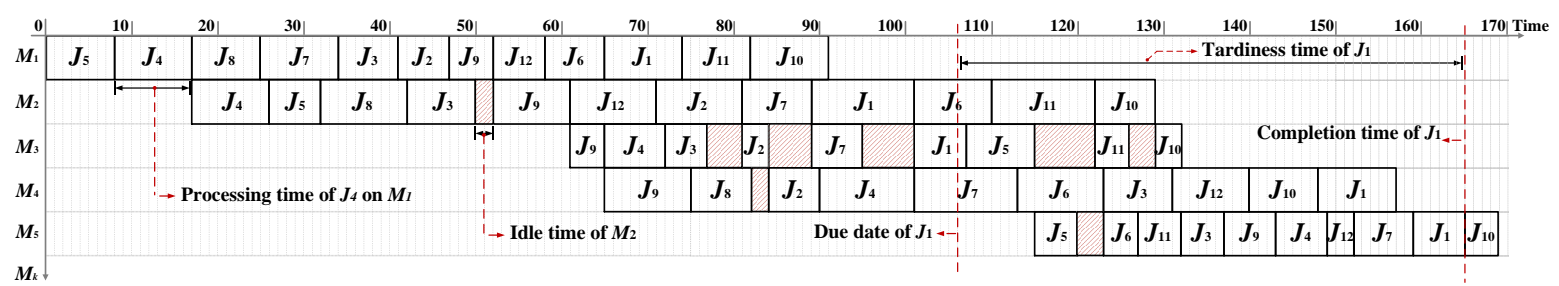

Figure 7. The Gantt chart of the example solution.

Based on the basic data of this case and the job sequences of the aforementioned solution, the calculation of NPE and TF are demonstrated in Tables 5 and 6, respectively.

Table 5. The NPE on the machines in the given schedule.

\begin{tabular}{ccccccc}
\hline Machine & $\boldsymbol{M}_{1}$ & $\boldsymbol{M}_{2}$ & $\boldsymbol{M}_{3}$ & $\boldsymbol{M}_{4}$ & $\boldsymbol{M}_{5}$ & Total \\
\hline Idle time & 0 & 2 & 25 & 2 & 3 & 32 \\
NPE & 0 & 7 & 70 & 4.2 & 11.1 & 92.3 \\
\hline
\end{tabular}

Table 6. The TF of the jobs in the given schedule.

\begin{tabular}{cccccccccccccc}
\hline Job & $J_{1}$ & $J_{2}$ & $J_{3}$ & $J_{4}$ & $J_{5}$ & $J_{6}$ & $J_{7}$ & $J_{8}$ & $J_{9}$ & $J_{10}$ & $J_{11}$ & $J_{12}$ & Total \\
\hline TF & 295 & 0 & 68 & 10 & 0 & 30 & 200 & 0 & 0 & 112 & 0 & 0 & 715 \\
\hline
\end{tabular}

\subsection{Results Comparison and Discussion}

The non-dominated Sorting Genetic Algorithm (NSGA) and its related improved algorithms are widely applied in the multi-objective optimisation of workshop scheduling problems [37,38]. To assess the solutions' quality of MCEA, the NSGA is compared in this study with the input of case 3. The results of the MCEA and NSGA after 10 independent runs are shown in Figure 8 and they are mainly distributed in the two zones enclosed within the red dotted lines and yellow dotted lines, respectively. The intersection zone contains the results of both algorithms, as enclosed with the blue dotted lines. The computation times of the MCEA and NSGA are approximates, ranging from $150 \mathrm{~s}$ to $200 \mathrm{~s}$. The length of the computation time is mainly influenced by the parameters including the scale of the case, the iteration times, and the size of the population. As demonstrated in Figure 8 , the results of MCEA dominate those of NSGA. 


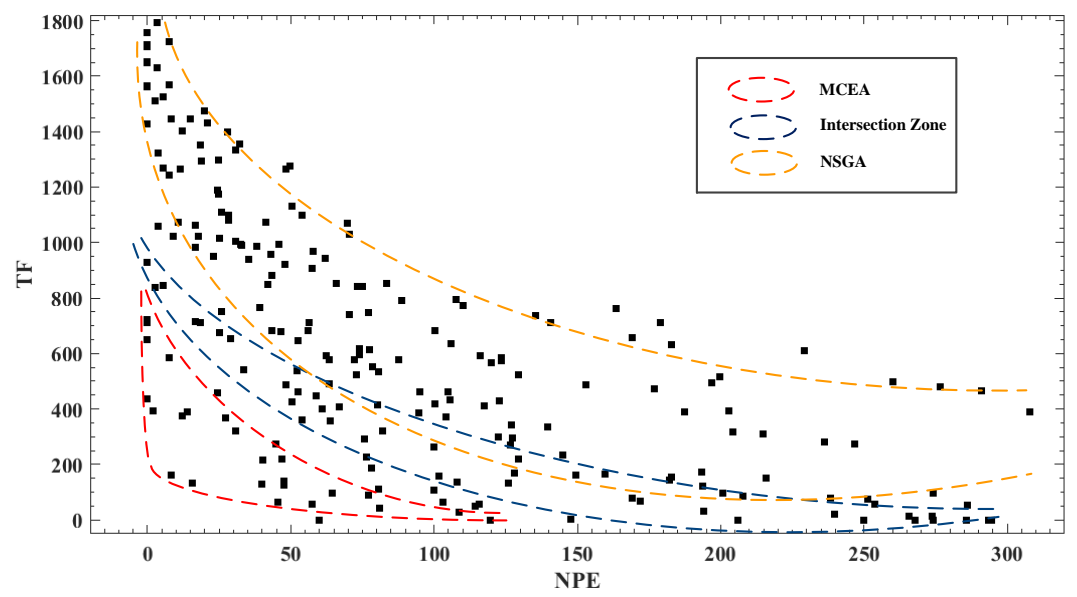

Figure 8. The results obtained by MECA and NSGA in case 3.

To verify the effectiveness of the MFSS, three traditional scheduling approaches are used in this case [39]. FCFS indicates first come first served and the jobs are processed in the sequence of appearance. SPT is the shortest process time first principle and it aims to shorten the processing time of the jobs. EDD represents the earliest due date first rule, which considers the earliest due date as a priority. In this study, all jobs are released at the same time and they are processed in the sequence of being received. The description and comparison of each approach are shown in Table 7.

Table 7. The comparison with three scheduling approaches.

\begin{tabular}{cll}
\hline Approach & \multicolumn{1}{c}{ Description } & \multicolumn{1}{c}{ Result } \\
\hline \multirow{3}{*}{ MFSS } & & NPE: 0, TF: 438; NPE: 2.1, TF: 392; NPE: \\
& Using the proposed mathematic models & 8.4, TF: 163; NPE: 15.8, TF: 133; NPE: 39.9, \\
& and MCEA algorithm to schedule & TF: 130; NPE: 45.4, TF: 63; NPE: 57.4, TF: \\
& 55; NPE: 59.8, TF: 0 \\
\hline FCFS & First come first served in the queue & NPE: 314.7, TF: 0 \\
\hline SPT & Shortest process time first & NPE: 433, TF: 165 \\
\hline EDD & Earliest due date first & NPE: 324.1, TF: 0 \\
\hline
\end{tabular}

According to the table, the MFSS performs best in saving energy and decreasing TF. FCFS is relatively simple and accessible, while the randomness of the jobs' release sequence has a negative effect on the scheduling efficiency. Energy saving and time optimisation scheduling are difficult to achieve. SPT minimises the cycle time, while the due date and tardiness fine of the jobs are different. Scheduling without considering the variant urgency and importance of jobs is not efficient to reduce the tardiness and the total TF. EDD takes the due date into account, which contributes to the on-time delivery. While the energy consumption is not saved using this approach, based on the data in the chart and table, the MFSS solutions dominate those of FCFS, SPT, and EDD. The MFSS is an effective approach for obtaining optimal scheduling schemes that minimise the TF and NPE. TF can be controlled at a low value and about $69.97 \%$ of NPE can be reduced using the MFSS solutions compared to FCFS, SPT, and EDD.

\section{Conclusions and Future Work}

A mixed-flow shop scheduling problem derived from a customised manufacturing environment is described in this paper. In the mixed-flow shop scenario, jobs with both standard and customised processes are processed in a workshop. Three main difficulties of scheduling in this scenario are solved: (1) more uncertainties are brought to the machines in selecting the next job to process because of the difference of the jobs' process routes with the purpose of minimising the NPE and TF; (2) compared 
with a typical flow shop, the job sequence on each machine can be different, which causes a higher computational burden; (3) a conflict exist between the TF and NPE, thus the trade-off of the two objectives should be considered. We propose a scheduling approach to provide feasible schemes for decision-makers to minimise the NPE and TF in such scenarios. We developed a mathematic model to describe the effect of scheduling schemes on NPE and TF. Then, we proposed the heuristic algorithm MCEA to obtain the Pareto solutions by the trade-offs between NPE and TF. To validate its feasibility, we use the deterministic algorithm, DSB. A case study with 12 processing jobs on 5 machines has been conducted using the MFSS. The MCEA is compared with the NSGA and the dominant solutions of the MCEA are demonstrated in this scenario. The results of the MFSS have been compared with those of three traditional schedule approaches. By comparison, $69.97 \%$ of NPE can be reduced by using the MFSS and there is a $10 \%$ possibility to reduced TF to zero. This demonstrates the effectiveness of the proposed approach in reducing TF and NPE. This research has a range of limitations: for example, we made some assumptions of the same release and constant processing time that limit the versatility of our method. Additionally, the robustness and precision of the algorithm can be improved through testing with additional cases.

For future works, the stochastic arrival and the real processing time of the jobs should be taken into consideration so that the approach can react quickly to the dynamic and changing workshop. Besides, in practical production, the jobs and machines can be even larger. For the extrusion die workshop mentioned in the introduction, the daily yield required is as high as ninety. Thus, the algorithm needs to be improved to guarantee both the computing speed and effectiveness in practical large-scale scheduling cases. Finally, parallel machines and more factors including transportation and setup should be incorporated into the mathematical model to make the model more applicable to actual production.

Author Contributions: Conceptualization, C.P. and L.H.; Methodology, C.P.; Software, C.P. and Y.Z.; Formal Analysis, T.P.; Investigation, T.P.; Resources, R.T., T.P. and L.H.; Writing-Original Draft Preparation, C.P.; Writing-Review \& Editing, C.P. and T.P.; Supervision, R.T. and T.P.; Funding Acquisition, R.T. and T.P.

Funding: This research was funded by National Natural Science Foundation of China grant number No. 51805479 and No. U151248.

Acknowledgments: The authors would like to thank the researchers in the Institute of Industrial Engineering, Zhejiang University, for their inputs.

Conflicts of Interest: The authors declare no conflict of interest.

\section{Abbreviations and Nomenclature}

$\begin{array}{ll}\text { Abbreviations } & \begin{array}{l}\text { Description } \\ \text { the proposed Mixed-Flow Shop Scheduling approach } \\ \text { MFSS }\end{array} \\ \text { NPE } & \text { Non-Processing Energy } \\ \text { PE } & \text { Processing Energy } \\ \text { TF } & \text { Tardiness Fine } \\ \text { MEPF } & \text { Manufacturing Energy consumption of Production Facilities } \\ \text { MCEA } & \text { multi-objective Evolutionary Algorithm with Multi-Chromosome } \\ \text { NSGA-II } & \text { Non-dominated Sorting Genetic Algorithm II } \\ \text { DSB } & \text { Deterministic Search method with Boundary } \\ \text { NSGA } & \text { Non-dominated Sorting Genetic Algorithm } \\ \text { FCFS } & \text { First Come First Served in the queue } \\ \text { SPT } & \text { Shortest Process Time first } \\ \text { EDD } & \text { Earlies Due Date first }\end{array}$




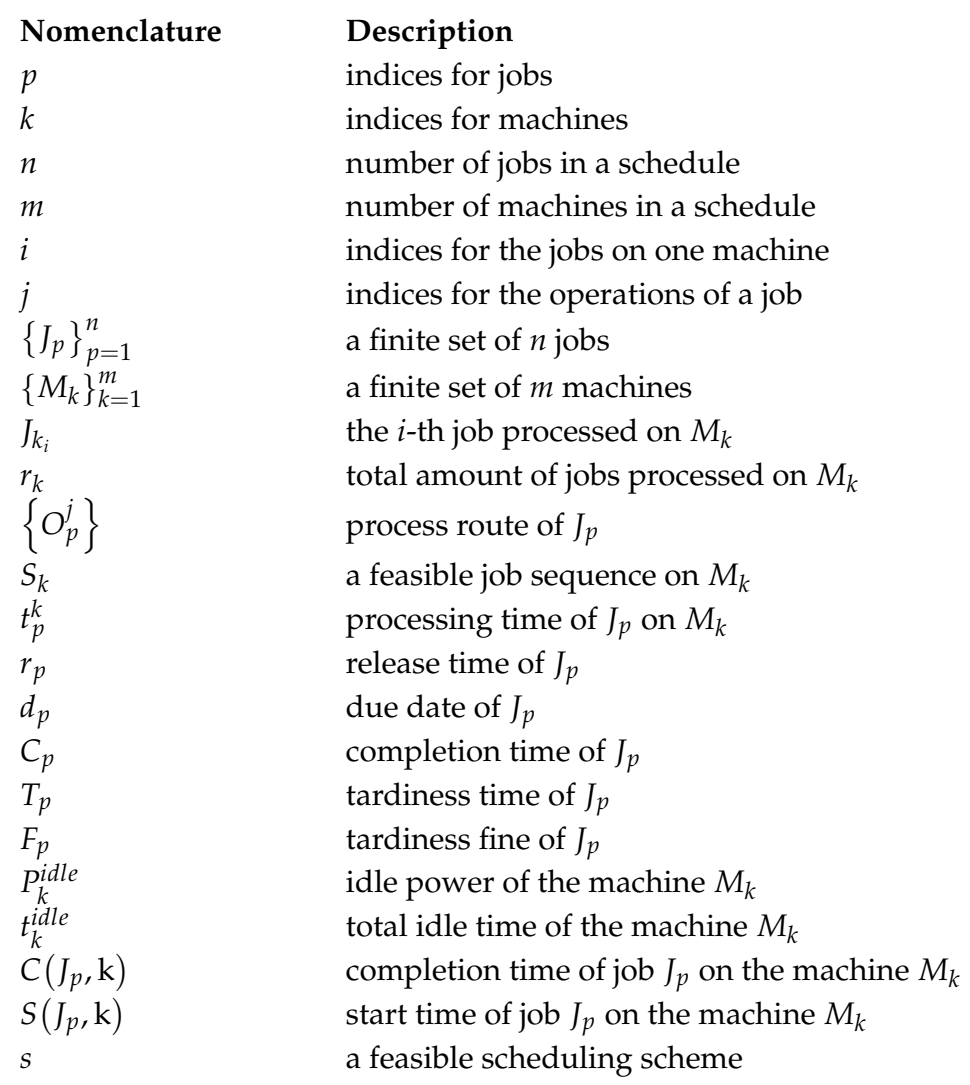

\section{Appendix A}

\begin{tabular}{|c|c|c|}
\hline Number & Model and Constrains & Description \\
\hline 1 & Multi $=$ Minimise $\{\mathrm{TF}(s), \operatorname{NPE}(s)\}$ & $\begin{array}{l}\text { Multi-objective function Multi is to minimise the NPE and } \\
\text { TF at the same time. }\end{array}$ \\
\hline 3 & $\operatorname{NPE}(s)=\sum_{k=1}^{m} P_{k}^{\text {idle }} \times t_{k}^{\text {idle }}$ & $\begin{array}{l}\text { Given a feasible scheduling scheme } s \text {, the total NPE NPE }(s) \\
\text { is the sum of each machine's NPE. }\end{array}$ \\
\hline 5 & $C_{p}=C\left(J_{p}, \max \left\{O_{p}^{j}\right\}\right)$ & $\begin{array}{l}\text { Completion time of } J_{p} \text { is the moment that its last operation } \\
\text { finishes. }\end{array}$ \\
\hline 6 & $t_{k}^{\text {idle }}=C\left(J_{k_{r_{i}}}, k\right)-S\left(J_{k_{1}}, k\right)-\sum_{i=1}^{r_{k}} t_{k_{i}}^{k}$ & $\begin{array}{l}\text { Total idle time of the machine } M_{k} \text { equals the completion } \\
\text { moment of its last job minus the start moment of its first job } \\
\text { minus the total processing time of all the jobs processed on } \\
\text { it. }\end{array}$ \\
\hline 8 & $\begin{array}{l}\operatorname{NPE}(s)= \\
\sum_{k=1}^{m}\left[\max \left\{C\left(J_{p}, k\right)\right\}-\min \left\{S\left(J_{p}, k\right)\right\}-\sum_{p=1}^{n} t_{p}^{k} X_{p k}\right] \times P_{k}^{\text {idle }}\end{array}$ & $\begin{array}{l}\text { Given a feasible scheduling scheme } s \text {, the total NPE NPE }(s) \\
\text { equals the sum of each machine's idle time multiply by its } \\
\text { idle power. }\end{array}$ \\
\hline 9 & $\sum_{p=1, k=1}^{p=n, k=m} X_{p k}=\left\{\begin{array}{cc}1, \exists O_{p}^{j} \in\left\{O_{p}^{j}\right\}=k \\
0, & \text { else }\end{array}\right.$ & $\begin{array}{l}\text { Restrict the jobs to process according to the process routes. If } \\
\text { one procedure of } J_{p} \text { is on } M_{k} \text {, then } X_{p k}=1 \text {. No job need use } \\
\text { one machine twice. }\end{array}$ \\
\hline 10 & $C\left(J_{k_{i}}, k\right) \geq C\left(J_{k_{i-1}}, k\right)+t_{k_{i}}^{k}, i \in\left(2, r_{k}\right) ; k_{i} \in\left(2, r_{k}\right)$ & $\begin{array}{l}\text { Define that only one job can be processed on a machine at } \\
\text { the same time and all processes are non-preemptive. }\end{array}$ \\
\hline 11 & $C\left(J_{p}, O_{p}^{j}\right) \geq C\left(J_{p}, O_{p}^{j-1}\right)+t_{p}^{O_{p}^{j}}, p \in(1, n) ; j \in(2, k) ; O_{p}^{j} \in(1, k)$ & $\begin{array}{l}\text { Guarantee that the job cannot be ceased machining unless } \\
\text { the whole job has been finished }\end{array}$ \\
\hline
\end{tabular}




\section{References}

1. Hu, L.; Peng, C.; Evans, S.; Peng, T.; Liu, Y.; Tang, R.; Tiwari, A. Minimising the machining energy consumption of a machine tool by sequencing the features of a part. Energy 2017, 121, 292-305. [CrossRef]

2. Administration UEI. Annual Energy Review; Government Printing Office: Washington, DC, USA, 2011.

3. Jia, S.; Yuan, Q.; Lv, J.; Liu, Y.; Ren, D.; Zhang, Z. Therblig-embedded value stream mapping method for lean energy machining. Energy 2017, 138, 1081-1098. [CrossRef]

4. Chen, G.; Zhang, L.; Arinez, J.; Biller, S. Energy-efficient production systems through schedule-based operations. IEEE Trans. Autom. Sci. Eng. 2013, 10, 27-37. [CrossRef]

5. He, Y.; Li, Y.; Wu, T.; Sutherland, J.W. An energy-responsive optimization method for machine tool selection and operation sequence in flexible machining job shops. J. Clean. Prod. 2015, 87, 245-254. [CrossRef]

6. Kordonowy, D.N. A Power Assessment of Machining Tools; Massachusetts Institute of Technology: Cambridge, MA, USA, 2002.

7. Wiendahl, H.-P. Load-Oriented Manufacturing Control; Springer: Berlin/Heidelberg, Germany, 1995.

8. Pinedo, M.L. Scheduling: Theory, algorithms, and Systems; Springer: New York, NY, USA, 2016.

9. Liu, Y.; Dong, H.; Lohse, N.; Petrovic, S.; Gindy, N. An investigation into minimising total energy consumption and total weighted tardiness in job shops. J. Clean. Prod. 2014, 65, 87-96. [CrossRef]

10. Lu, C.; Gao, L.; Li, X.; Pan, Q.; Wang, Q. Energy-efficient permutation flow shop scheduling problem using a hybrid multi-objective backtracking search algorithm. J. Clean. Prod. 2017, 144, 228-238. [CrossRef]

11. Bierwirth, C.; Kuhpfahl, J. Extended grasp for the job shop scheduling problem with total weighted tardiness objective. Eur. J. Oper. Res. 2017, 261, 835-848. [CrossRef]

12. May, G.K.; Stahl, B.; Taisch, M.; Prabhu, V. Multi-objective genetic algorithm for energy-efficient job shop scheduling. Int. J. Prod. Res. 2015, 53, 7071-7089. [CrossRef]

13. Yildirim, M.B.; Mouzon, G. Single-machine sustainable production planning to minimize total energy consumption and total completion time using a multiple objective genetic algorithm. IEEE Trans. Eng. Manag. 2012, 59, 585-597. [CrossRef]

14. Shrouf, F.; Ordieres-Meré, J.; García-Sánchez, A.; Ortega-Mier, M. Optimizing the production scheduling of a single machine to minimize total energy consumption costs. J. Clean. Prod. 2014, 67, 197-207. [CrossRef]

15. Yin, L.; Li, X.; Lu, C.; Gao, L. Energy-efficient scheduling problem using an effective hybrid multi-objective evolutionary algorithm. Sustainability 2016, 8, 1268. [CrossRef]

16. Li, Z.; Yang, H.; Zhang, S.; Liu, G. Unrelated parallel machine scheduling problem with energy and tardiness cost. Int. J. Adv. Manuf. Technol. 2015, 84, 213-226. [CrossRef]

17. Moon, J.-Y.; Park, J.; Shin, K. Optimization of production scheduling with time-dependent and;machine-dependent electricity cost for industrial energy efficiency. Int. J. Adv. Manuf. Technol. 2013, 68, 523-535. [CrossRef]

18. Ding, J.Y.; Song, S.; Zhang, R.; Chiong, R.; Wu, C. Parallel machine scheduling under time-of-use electricity prices: New models and optimization approaches. IEEE Trans. Autom. Sci. Eng. 2016, 13, 1138-1154. [CrossRef]

19. Zhang, H.; Zhao, F.; Fang, K.; Sutherland, J.W. Energy-conscious flow shop scheduling under time-of-use electricity tariffs. CIRP Ann.-Manuf. Technol. 2014, 63, 37-40. [CrossRef]

20. Liu, Y.; Dong, H.; Lohse, N.; Petrovic, S. A multi-objective genetic algorithm for optimisation of energy consumption and shop floor production performance. Int. J. Prod. Econ. 2016, 179, 259-272. [CrossRef]

21. Zhang, R.; Chiong, R. Solving the energy-efficient job shop scheduling problem: A multi-objective genetic algorithm with enhanced local search for minimizing the total weighted tardiness and total energy consumption. J. Clean. Prod. 2015, 112, 3361-3375. [CrossRef]

22. Tang, D.; Dai, M.; Salido, M.A.; Giret, A. Energy-efficient dynamic scheduling for a flexible flow shop using an improved particle swarm optimization. Comput. Ind. 2016, 81, 82-95. [CrossRef]

23. Dai, M.; Tang, D.; Giret, A.; Salido, M.A.; Li, W.D. Energy-efficient scheduling for a flexible flow shop using an improved genetic-simulated annealing algorithm. Robot. Comput.-Integr. Manuf. 2013, 29, 418-429. [CrossRef]

24. Li, X.; Xing, K.; Wu, Y.; Wang, X.; Luo, J. Total energy consumption optimization via genetic algorithm in flexible manufacturing systems. Comput. Ind. Eng. 2017, 104, 188-200. [CrossRef] 
25. Mouzon, G.; Yildirim, M.B.; Twomey, J. Operational methods for minimization of energy consumption of manufacturing equipment. Int. J. Prod. Res. 2007, 45, 4247-4271. [CrossRef]

26. Zhang, L.; Li, X.; Gao, L.; Zhang, G. Dynamic rescheduling in fms that is simultaneously considering energy consumption and schedule efficiency. Int. J. Adv. Manuf. Technol. 2013, 87, 1-13. [CrossRef]

27. Liu, X.; Zou, F.; Zhang, X. Mathematical model and genetic optimization for hybrid flow shop scheduling problem based on energy consumption. In Proceedings of the Control and Decision Conference CCDC2008, Yantai, China, 2-4 July 2008; pp. 1002-1007.

28. Tong, Y.; Li, J.; Li, S.; Li, D. Research on energy-saving production scheduling based on a clustering algorithm for a forging enterprise. Sustainability 2016, 8, 136. [CrossRef]

29. Li, X.; Lu, C.; Gao, L.; Xiao, S.; Wen, L. An Effective Multi-Objective Algorithm For Energy Efficient Scheduling In A Real-Life Welding Shop. In IEEE Transactions on Industrial Informatics; IEEE: Piscataway, NJ, USA, 2018.

30. Zhou, D.C.; Zeng, L. Intelligent scheduling method oriented to multi-varieties and small-batch production mode. In Applied Mechanics and Materials; Trans Tech Publications: Zurich, Switzerland, 2013; pp. 1269-1274.

31. Huang, T.-C.; Lin, B.M. Batch scheduling in differentiation flow shops for makespan minimisation. Int. J. Prod. Res. 2013, 51, 5073-5082. [CrossRef]

32. Wang, M.; Zhong, R.Y.; Dai, Q.; Huang, G.Q. A mpn-based scheduling model for iot-enabled hybrid flow shop manufacturing. Adv. Eng. Inform. 2016, 30, 728-736. [CrossRef]

33. Huang, H.-H.; Pei, W.; Wu, H.-H.; May, M.-D. A research on problems of mixed-line production and the re-scheduling. Robot. Comput.-Integr. Manuf. 2013, 29, 64-72. [CrossRef]

34. Zitzler, E.; Knowles, J.; Thiele, L. Quality assessment of pareto set approximations. In Multiobjective Optimization; Springer: Berlin, Germany, 2008; pp. 373-404.

35. Auger, A.; Bader, J.; Brockhoff, D.; Zitzler, E. Hypervolume-based multiobjective optimization: Theoretical foundations and practical implications. Theor. Comput. Sci. 2012, 425, 75-103. [CrossRef]

36. Hu, L.; Tang, R.; Liu, Y.; Cao, Y.; Tiwari, A. Optimising the machining time, deviation and energy consumption through a multi-objective feature sequencing approach. Energy Convers. Manag. 2018, 160, 126-140. [CrossRef]

37. Mousavi, S.; Mahdavi, I.; Rezaeian, J.; Zandieh, M. An efficient bi-objective algorithm to solve re-entrant hybrid flow shop scheduling with learning effect and setup times. Oper. Res. 2018, 18, 123-158. [CrossRef]

38. Khan, B.; Hanoun, S.; Johnstone, M.; Lim, C.P.; Creighton, D.; Nahavandi, S. Multi-objective job shop scheduling using i-nsga-iii. In Proceedings of the 2018 Annual IEEE International Systems Conference (SysCon), Vancouver, BC, Canada, 24-26 April 2018; pp. 1-5.

39. Faccio, M.; Nedaei, M.; Pilati, F. A comparative analysis of job scheduling for optimum performance of parallel machines by considering the energy consumption. Eur. J. Eng. Res. Sci. 2018, 3, 6-11. [CrossRef] 